\title{
À cheval sur les traditions... Tourisme et cérémoniels équestres en transformation : le cas de l'Italie
}

Riding traditions... Tourism and equestrian ceremonials in transformation: the case of Italy

\section{Letizia Bindi}

\section{OpenEdition \\ Journals}

Édition électronique

URL : http://journals.openedition.org/tourisme/3106

DOI : $10.4000 /$ tourisme.3106

ISSN : 2492-7503

Éditeur

Éditions touristiques européennes

\section{Référence électronique}

Letizia Bindi, « À cheval sur les traditions... Tourisme et cérémoniels équestres en transformation : le cas de l'Italie », Mondes du Tourisme [En ligne], 18 | 2020, mis en ligne le 01 mars 2021, consulté le 15 mars 2021. URL : http://journals.openedition.org/tourisme/3106 ; DOI : https://doi.org/10.4000/

tourisme.3106

Ce document a été généré automatiquement le 15 mars 2021.

\section{cc) (1)}

Mondes du tourisme est mis à disposition selon les termes de la licence Creative Commons Attribution - Pas d'Utilisation Commerciale - Pas de Modification 4.0 International. 


\section{À cheval sur les traditions... Tourisme et cérémoniels équestres en transformation : le cas de l'Italie}

Riding traditions... Tourism and equestrian ceremonials in transformation: the case of Italy

Letizia Bindi

\section{Introduction}

1 L'Italie est riche d'un ensemble de pratiques traditionnelles festives caractérisées par la présence et l'implication d'animaux - en particulier d'équidés, mais pas exclusivement -, qui ont été fortement contestées dans la sphère publique et politique italienne au cours de la décennie passée, principalement en raison de questions de droits des animaux (Bindi, 2012).

Cette contribution se propose de réfléchir à l'évolution des cérémonies traditionnelles équestres italiennes, confrontées au double phénomène de leur médiatisation internationale et de leur remise en question par le droit animalier. Nous discuterons, plus particulièrement, leur relation complexe à leur mise en tourisme, qui participe à leur redéfinition, en intégrant les nouvelles sensibilités. Les résultats s'appuient sur des cas particuliers observés et documentés au cours d'une recherche ethnographique.

L'un des terrains emblématiques pour cette recherche a été le Palio di Siena (Palio de Sienne) - célèbre cérémonial, connu dans le monde entier, qui est fortement contesté chaque année par les mouvements pour les droits des animaux en raison des blessures ou de la mort, dans certains cas, des chevaux impliqués. L'observation se fonde aussi sur d'autres Palii et compétitions équestres traditionnelles, tout aussi anciennes et cruciales pour les communautés en fête (Asti, Legnano, Oristano, etc.) qui ont subi le même sort que le Palio de Sienne. Enfin, un cas tout particulier est celui des Carresi du Bas Molise, au cours desquels, en plus des chevaux en compétition pour la dévotion des 
communautés locales, des bœufs attelés participent aussi à des courses similaires. Or, ces Carresi ont été suspendus à deux reprises au cours des cinq dernières années en raison de controverses sur les droits des animaux, auxquelles se sont ajoutées des considérations sécuritaires liées à la gestion d'événements présentant un taux de concentration élevé du public.

Dans tous ces cas, l'une des questions les plus importantes posées par les communautés touchées par ces controverses sur les droits des animaux (Noske, 1997 ; Ballacchino et Bindi, 2017) a été de savoir si la plus grande visibilité médiatique et la mondialisation qui avaient conduit, par le passé, à l'émergence de ces pratiques sur la scène nationale puis internationale - ont été, ou non, à l'origine de l'attaque des mouvements des droits animaux (Mugnaini, 2013; Ballacchino et Bindi, 2014; Meloni, 2014). À travers mes recherches bibliographiques sur le Palio de Sienne, mon observation partielle de ce terrain d'investigation et, plus encore, au cours de mon ethnographie des Carresi de chars à bœufs du Molise, j'ai constaté que les habitants de ces villes et villages ainsi que les participants à ces manifestations traditionnelles compétitives ont déclaré craindre la progressive médiatisation des cérémoniels, la considérant comme une menace. Ils considèrent que cette visibilité croissante a déterminé l'émergence des attaques des mouvements pour les droits des animaux et, plus généralement, des réactions critiques d'une partie du public qui assiste à ces événements, à distance ou à travers des médias (télévision, réseaux sociaux, etc.).

5 En parallèle, une autre question soulevée par ces études de cas est le dilemme entre les droits des animaux, soutenus avec force et vivacité par les mouvements de défense des droits des animaux et leurs dirigeants politiques, et les droits des communautés à maintenir leurs pratiques traditionnelles. En effet, ces dernières revendiquent leur droit de perpétuer des usages culturels, mais aussi de les promouvoir comme autant de patrimoines immatériels à même de représenter des leviers de développement touristique territorial.

$6 \quad$ Les différends théoriques et juridiques soulevés par les mouvements de défense des droits des animaux mettent en exergue, comme élément central de cette reconsidération critique, la question de la représentation, au travers de ce que Rabinow (1992) a appelé, en accord avec Michel Foucault, la «biopolitique», voire la "biosociale» (Helmreich, 2009). La question du spécisme pose, en effet, une question de contrôle et de pouvoir sur le corps animal, une considération critique et radicale de la souffrance et de la constriction que les hommes exerceraient sur les sujets animaux réduits à de purs objets, réifiés par l'emploi productif et/ou spectaculaire, comme moyens pour une compétition cérémonielle considérée par les mouvements animalistes comme atavique et brutale.

7 Cela soulève, de plus, la question tout aussi fondamentale de la " voix », que Appadurai développe $(1988$, p. 17) en discutant des sujets de la communication et du témoignage : " parler ", " parler pour/à la place de », et qui dans les relations homme/animal semble désormais devenir de plus en plus cruciale et inaliénable. Reconsidéré et reformulé à la lumière de ces contributions, l'anthropologie finit par développer un nouvel objectif, parfaitement résumé par Kohn :

8 Le but dans l'ethnographie multi-espèces ne devrait pas seulement être de donner une voix, une représentation ou une subjectivité au non-humain pour les reconnaître comme « autres », identifiables dans leur différence - mais pour nous forcer à repenser 
radicalement ces catégories de notre analyse, car elles concernent tous les êtres. (cité par Kirskey et Helmreich, 2010)

9 Cette approche nécessite une méthodologie hybride, qui implique la déconstruction des objets et des modes anthropocentriques afin de faciliter des relations plus égales avec les animaux. En effet, ces derniers ne doivent plus être compris comme des produits de la pratique sociale humaine, mais plutôt comme des sujets autonomes capables d'interagir avec les sociétés. Ce faisant, nous pouvons échapper aux visions des relations homme-homme et homme-animal caractérisées essentiellement par un métanarratif anthropocentrique et scientifique, par un paradigme de domination qui tourne autour des hiérarchies, du contrôle et de l'exploitation et ne prend pas en compte les échanges intersubjectifs ni les formes de coopération (Davis, Maurstad et Cowles, 2013 ; Balcombe, 2009).

Dans le même temps, cependant, les mouvements de défense des droits des animaux ont exprimé des critiques et des préoccupations au sujet de ces pratiques cérémoniales, les percevant comme une menace pour le bien-être des animaux et soulevant des questions juridiques entourant la protection de ce droit fondamental et la défense de la subjectivité animale (Ryder, 1989).

11 Le but de ma recherche est, d'une part, d'observer comment les débats animaliers utilisent cette vision binaire d'opposition homme-animal et comment ils ont impacté les déroulements des cérémoniels équestres; et, d'autre part, d'analyser le rôle du tourisme dans la recodification de ces évènements, entre résistances et intégration des sensibilités nouvelles, générant d'importantes transformations socio-culturelles.

12 J'ai donc essayé d'aborder ces questions à travers une analyse attentive de la littérature critique produite sur ces occurrences, en particulier, pour ce qui concerne, le Palio de Sienne, en collectant les travaux précédemment édités : j'ai parcouru la littérature historique, anthropologique et sociologique produite surtout dans les trois dernières décennies sur la valeur du Palio en termes identitaires et en tant qu'enjeu patrimonial et touristique formidable pour la ville et la communauté siennoise. Il s'agit d'une analyse de textes et de vidéo-documents qui traitent des débats surgis récemment au sujet des polémiques animalistes. Ces courants de défense animale ont tout d'abord demandé la suppression du Palio, puis une profonde modification de la pratique rituelle compétitive pour qu'elle puisse perdurer.

13 Concernant les Carresi du Bas Molise (courses de chariots à bœufs), les données produites pendant mon ethnographie à travers la méthodologie "classique » de type ethnographique, comprennent :

- Des observations participantes : pendant la préparation des courses et pendant les courses ; de la vie quotidienne des groupes de participants avec leurs animaux aux écuries; des représentations de ces courses auprès du public présent au cours des compétitions ou des épreuves ;

- De longs entretiens qualitatifs individuels avec des membres des Partiti des différents cérémoniels et des communautés qui assistent aux rituels; des entretiens avec les administrateurs, les vétérinaires, les responsables de la sécurité publique au cours des fêtes ;

- Des productions de vidéo-documentation des activités ; analyse des vidéos avec les témoins et les acteurs locaux et photo-élicitation d'images relatives aux courses et leurs commentaires ;

- Des focus group avec des représentants des écuries et des familles qui se défient dans les compétitions équestres. 

utilisé la méthodologie de l'« enclicage » comme forme d'insertion du chercheur dans une société à travers des groupes particuliers, des réseaux spécifiques. Cette méthodologie est redoutable autant qu'inévitable et implique un certain risque d'assimilation, mais aussi une complicité "à une "clique" ou une "faction" locale» (Olivier de Sardan, 1995, p. 81). Pour ces raisons, cette méthodologie a été critiquée par certains ethnographes et anthropologues, pointant les risques de partialité du chercheur, due à un centrage exclusif sur un groupe particulier. J'utilise au contraire le terme "enclicage" en reprenant certaines considérations de chercheurs qui ont travaillé sur l'anthropologie appliquée et l'engagement dans les sciences sociales. Dans un contexte de débats et de fortes tensions, comme c'est le cas dans mon étude, il est nécessaire de prendre officiellement parti, afin de pouvoir être admis au sein des groupes étudiés et de participer à leurs activités privées, voire secrètes.

Cet ensemble de techniques d'enquête m'a permis d'observer des moments particuliers et de comprendre des aspects très intimes des fêtes, ce que je n'aurais pas pu réaliser avec une méthodologie plus détachée et non partisane. différentes personnes interrogées à travers leur implication dans la préparation de ces compétitions cérémonielles ; les valeurs culturelles et dévotionnelles qu'ils y associent ; leur regard sur les pressions animalistes et leurs conséquences; leur vision de la mise en tourisme de ces événements et ses enjeux.

La vidéo-documentation a permis d'analyser la manière dont se répartissent, pendant les courses, les touristes et les membres de la communauté sur la scène des fêtes; et la façon dont ils sont plus ou moins inclus dans les moments les plus importants des rituels. En même temps, une attention particulière a été portée à la façon dont les médias appréhendent ces formes cérémonielles, ce qui nous a permis de comprendre l'influence des représentations médiatiques sur ces pratiques rituelles compétitives (Meloni, 2014 ; Ballacchino et Bindi, 2014).

\section{Des courses équestres rituelles confrontées aux sensibilités contemporaines}

\section{Le heurt des courses rituelles au droit animalier et à sa récupération politique}

Le Palio de Sienne est une course de chevaux qui a lieu deux fois par an, les 2 juillet et 16 août, à Sienne, en Italie. Dix chevaux et cavaliers, à cru et vêtus des couleurs de leur quartier, représentent dix des dix-sept Contrade ou quartiers de la ville ${ }^{1}$.

Le Palio organisé le 2 juillet porte le nom de Palio di Provenzano, en l'honneur de la Vierge de Provenzano. C'est une dévotion à la Vierge propre à Sienne, qui s'est développée autour d'une icône du Terzo (Quartier) Camollia en comméroration de la guérison des citoyens d'une épidémie de peste. Le Palio qui se tient le 16 août est nommé Palio de l'Assunta, en l'honneur de l'Assomption de Marie. Parfois, en cas d'événements exceptionnels ou d'anniversaires locaux ou nationaux particulièrement importants et jugés pertinents, la communauté de la ville peut décider de célébrer un Palio extraordinaire, organisé entre mai et septembre. Le dernier Palio extraordinaire a 
été organisé pour célébrer le centenaire de la fin de la Première Guerre mondiale en 2018 (Silverman, 1979 ; Falassi, 1980 ; Simonicca, 1990 ; Civai et Tosi, 1995 ; Dundes et Falassi, 1975, 2005 ; Drechsler, 2006 ; Sensi et Santini, 1995-2002).

Un important Corteo Storico (un défilé historique en costume avec des spectacles de drapeaux, des joueurs de trompettes militaires médiévales, des dames et messieurs représentant la noblesse siennoise ancienne, etc.) précède la course, attirant nombre de spectateurs locaux et de touristes internationaux (Grassi, 1972 ; Heywood, 1993).

La Piazza del Campo, place sur laquelle se déroule l'événement, est couverte par une épaisse couche de terre. La course ne dure généralement pas plus de 90 secondes. Il est courant que quelques-uns des jockeys soient éjectés de leurs chevaux à l'occasion de virages périlleux. Il n'est pas rare non plus de voir des chevaux, non montés, terminer la course sans leurs jockeys et parfois même gagner, la présence du cavalier sur leur dos n'étant pas obligatoire pour les déclarer vainqueurs (AA.VV., 2003 ; AA.VV., 2004 ; Betti et Falassi, 2003)!

Illustration 1. Le Palio de Sienne, une course traditionnelle rituelle sur la place centrale del Campo

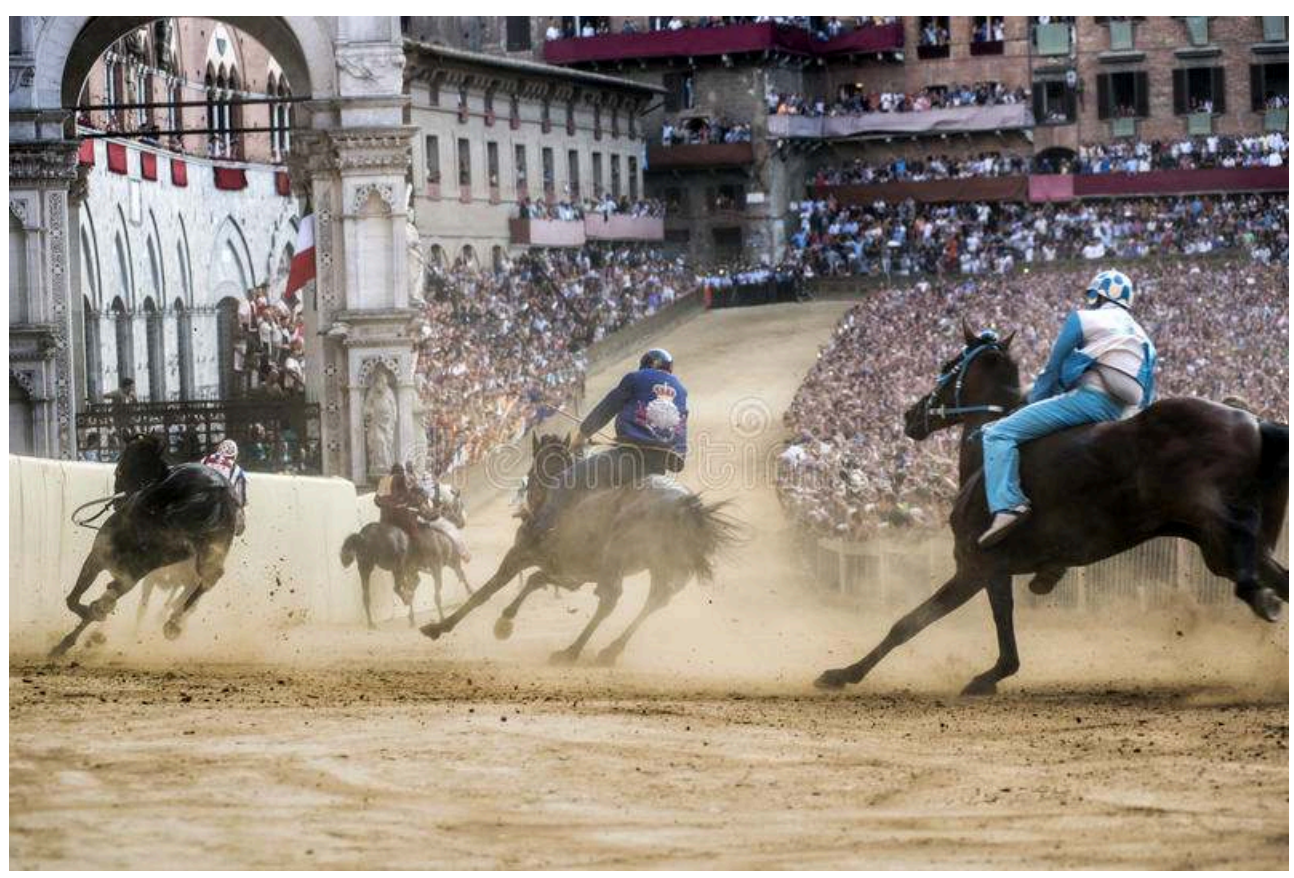

ID 59938695 @ M. Rohana | Dreamstime.com

En 2010, l'administration municipale de Sienne, soutenue par le Priorato des Contrade (l'organisme qui coordonne toutes les activités préparatoires au Palio), et l'Université de Sienne se sont investies dans la préparation d'un dossier de candidature du Palio de Sienne à la liste du patrimoine immatériel de l'Unesco. L'engagement local et régional étant très fort, ils pensaient que cette initiative serait couronnée de succès, mais cela n'a pas été pas le cas. En effet, en 2011, ils ont assisté à la récusation de la candidature du Palio à la liste du patrimoine immatériel de l'Unesco de la part du Comité interministériel italien, en dépit de l'important rayonnement médiatique de la cérémonie, de l'impact touristique de l'évènement et de son importance patrimoniale et historique pour la communauté. Cette récusation a résulté en une forte controverse opposant la municipalité et la ville de Sienne ainsi que la communauté perpétuant la pratique cérémoniale, d'une part, et le gouvernement national, d'autre part (Broccolini, 
2012). Ce conflit s'est plus particulièrement cristallisé en la personne de Madame Brambilla, sénatrice du parti de Forza Italia en 2010 lors de la candidature du Palio, devenue ministre du gouvernement de Silvio Berlusconi en 2011. Tous deux sont, en effet, très engagés dans la lutte des mouvements pour les droits des animaux, qui représentaient et représentent encore aujourd'hui une part importante de l'opinion publique. Ils constituent ainsi un électorat que Berlusconi avait le projet d'attirer politiquement, dans sa volonté de rassembler les partis de la Droite. Ainsi, M. Gianluca Felicetti, président de l'une des associations les plus actives pour les droits des animaux (la LAV, Ligue antivivisection), proche des positions idéologiques de la ministre Brambilla, déclarait en 2019 :

\begin{abstract}
Au Palio de Sienne pèse le poids d'au moins 48 chevaux décédés depuis 1970 et une enquête en cours permettra de faire la lumière sur les soupçons de dopage de certains chevaux. Ce sont des faits sérieux et dramatiques pour les animaux qui ont perdu la vie depuis que ce concours existe, et ils entachent l'image de l'une des plus belles villes du monde, dont le patrimoine architectural figure à juste titre sur la liste de l'Unesco. Il existe des alternatives pour préserver le concours et le festival, comme Lodi et d'autres villes qui depuis longtemps ont judicieusement choisi de remplacer les chevaux ou d'autres animaux par des chariots poussés par des hommes. C'est un exemple admirable de la possibilité de respecter véritablement les droits de tous [...]. La honte des chevaux morts et blessés ne permet pas au Palio de Sienne d'être un symbole positif pour l'Italie. (cité par Adinolfi, 2017) ${ }^{2}$
\end{abstract}

Le débat soulevé par ces polémiques a permis d'interroger la capacité du Palio de Sienne à être un « symbole national » du patrimoine immatériel. Il a, de plus, relancé le projet intéressant, et néanmoins controversé, de créer un registre des pratiques festives et cérémoniales mineures connues non seulement au niveau national, mais aussi international, grâce à la diffusion par des émissions des compétitions rituelles au niveau mondial. Il interpelle également sur la coexistence de pratiques traditionnelles, qui reposent sur l'utilisation des animaux, et de nouvelles sensibilités, soucieuses de leur droit et de leur bien-être, qui ne peuvent accepter l'existence de pratiques rituelles à risques pour ces « êtres sensibles ».

\title{
Des enjeux culturels mais aussi socioéconomiques complexes
}

Les mouvements pour les droits des animaux accusent ainsi le Palio d'être une pratique de «sauvages" qu'il faut supprimer. Ils ont tenté plusieurs fois d'interrompre la course, de l'interdire et de bloquer les entraînements des chevaux. Et ils se sont, bien évidemment, opposés à la candidature auprès de l'Unesco.

Pourtant, les enjeux socioéconomiques associés au Palio sont importants pour Sienne. D'une part, il est profondément associé au fonctionnement communautaire de la ville. Il cristallise l'appartenance à un lieu et à une tradition partagée. Il illustre, aussi, la réarticulation d'une relation homme-animal qui est d'abord une manière de penser un rapport entre culture et nature, mais aussi entre ville et campagne, en favorisant la rencontre d'un style de vie urbain - intégrant un rapport singulier au temps et aux loisirs - et d'un mode de vie et de travail lié aux sociétés rurales - notamment à travers l'élevage et l'entraînement des chevaux.

De plus, le Palio est devenu depuis longtemps un évènement international qui a contribué à faire de Sienne une destination touristique mondiale, figurant parmi les plus importantes villes d'art et de traditions européennes. Selon la chambre de 
commerce de Sienne Arezzo, les arrivées dans la province ont dépassé 2 millions de touristes en 2018, avec une augmentation de 7,9\% par rapport à 2017. Les nuitées se sont élevées à 5,3 millions, enregistrant une croissance de 6,7 \% par rapport à 2017. La durée moyenne de séjour reste sensiblement inchangée par rapport à 2017, autour de 2,6 jours. La fréquentation domestique et internationale de la région de Sienne continue de croître. Les touristes étrangers proviennent principalement des États-Unis, d'Allemagne, de France, de Chine, du Royaume-Uni et des Pays-Bas, qui restent des visiteurs fidèles. Les mois d'été, avec le pic de fréquentation de la période comprise entre juillet et août, couvrant les deux dates du Palio, représentent à eux seuls $43 \%$ de la fréquentation touristique de la ville ${ }^{3}$.

Le refus du gouvernement italien de présenter le dossier de candidature du Palio à la liste du patrimoine immatériel de l'Unesco a néanmoins eu des conséquences importantes sur la scène internationale, en suscitant une polémique. Il a également provoqué beaucoup de débats dans la ville de Sienne, interrogeant la population locale sur la construction et la gestion de Sienne en tant que destination touristique. Pourtant, dans les faits, cela n'a pas engendré de baisse de la fréquentation touristique, qu'elle soit domestique ou internationale, ce qui questionne aussi le rapport entre manifestation d'oppositions et popularité d'un évènement culturel.

Cette situation conflictuelle a, cependant, imposé à la communauté de faire évoluer les pratiques, en intégrant de nouvelles règles, plus particulièrement liées à : la gestion du fond de piste de la course aménagé sur l'historique Piazza del Campo ; la prévention des accidents ; la sécurité des chevaux à l'entraînement ; mais aussi la sécurité du public et des touristes qui assistent à la manifestation et qui sont déterminants dans la progressive transformation de la cérémonie traditionnelle en un évènement promotionnel et touristique. Par ailleurs, si les touristes sont fondamentaux, tant d'un point de vue économique que culturel, dans le processus de co-construction du Palio d'aujourd'hui, ils posent, néanmoins, le problème de la cohabitation, parfois délicate, de milliers de visiteurs et des populations locales participant au rituel. Leurs pratiques, motivations et représentations relèvent de logiques différenciées, notamment en lien à la relation à l'animal.

Aussi, afin de permettre d'assurer le présent et l'avenir du Palio en l'inscrivant dans une perspective durable, répondant à ces nouveaux enjeux complexes, la communauté a dû intégrer l'intervention de nouveaux acteurs, tels que des présidiums médicaux et vétérinaires pour contrôler la santé des hommes et des chevaux et éviter le dopage, mais aussi des agents de gestion et de contrôle des sols.

\section{Le tourisme, agent de co-constitution et de pérennisation des courses rituelles?}

\section{Le tourisme, acteur de la codification historique des courses rituelles}

Le tourisme a une action co-constituante complexe en matière de cultures équestres traditionnelles. Ainsi, le Palio est, à Sienne comme dans d'autres villes italiennes, un cérémoniel très ancien, dont on trouve des témoignages artistiques et documentaires historiques dans toutes les localités où il est célébré. 


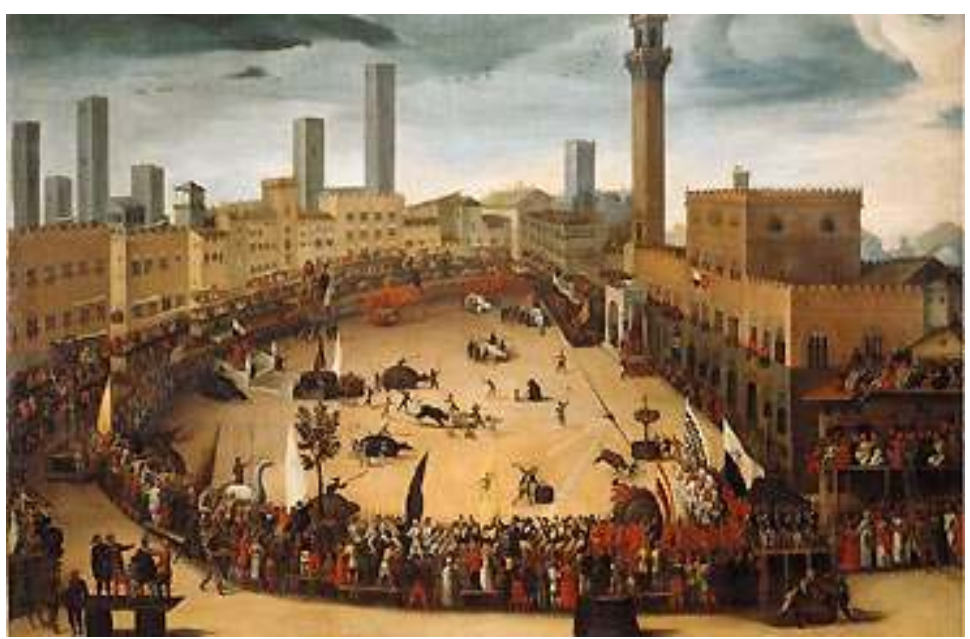

Monte dei Paschi di Siena (www.mps.it). Euvre conservée au Museo San Donato, Sienne. PD-Art

Néanmoins, c'est essentiellement au $x^{\mathrm{e}}$ siècle que les usages de ces cérémoniels ont été codifiés, dans un objectif de valorisation touristique. Ainsi, les règles du Palio de Legnano, en Lombardie, ont été déterminées dans les années 1930, à l'instar de plusieurs autres compétitions équestres locales d'origine médiévale, afin d'accroître leur lisibilité et leur attractivité touristique. Le Palio de Sienne, qui est devenu emblématique de la pratique à l'échelle internationale, a été définitivement codifié, dans sa configuration actuelle, pendant le régime fasciste, grâce à l'intervention de l'Opera Nazionale Dopolavoro, qui aspirait à en faire un instrument de mise en valeur culturelle et touristique nationale.

Le tourisme a donc, historiquement, contribué à la construction contemporaine, au travers de leur fixation, de ces cultures équestres. Il n'en interdit pas moins l'investissement fort des communautés, pour lesquelles le sens de ces manifestations cérémonielles dépasse largement leurs retombées touristiques. Les courses demeurent associées à des compétitions rituelles adossées à des valeurs identitaires et sociales. Elles sont d'ailleurs accompagnées de dîners rituels, de processions particulières dans les chapelles de quartiers (Terzi) où l'on bénit les chevaux, ou de réunions de soutien des Contrade (quartiers). Les citoyens de Sienne affirment qu'ils célèbreraient le Palio même s'il n'y avait aucun touriste présent sur la place. Ainsi, la mise en tourisme des différents Palii, depuis les années 1930, n'a pas subverti leur signification culturelle et identitaire.

33 Si le tourisme est un agent de co-constitution historique des fêtes équestres ritualisées en Italie, il induit néanmoins de nécessaires adaptations, surtout dans un contexte d'essor international, afin de répondre à des demandes parfois contradictoires.

\section{S'adapter pour perdurer : le tourisme, vecteur de compromis entre culture locale et attentes internationales?}

34 La perpétuation de ces courses traditionnelles équestres induit la nécessaire intégration de nouvelles sensibilités et normes. En première instance, la pression publique a été particulièrement forte en ce qui concerne le droit des animaux. Elle s'est 
illustrée par des manifestations, des sit-in, des actions parfois spectaculaires, par exemple des opposants s'enchaînant à l'entrée des parcours de compétitions rituelles, mais aussi des plaintes auprès des autorités judiciaires concernées, des demandes d'enquêtes antidopage, etc.

Ainsi, les compétitions sont dorénavant tenues de se dérouler dans des parcours validés par la Fédération équestre nationale, sur la base d'un règlement établi par la Fédération équestre internationale (FEI). Cela exclut donc tous les parcours rituels qui prévoient que les animaux courent dans les rues d'une ville ou d'un village, à moins qu'elles ne soient couvertes de sable selon le règlement. Elles doivent aussi se conformer à une série de précautions techniques qui n'étaient pas d'usage naguère dans ces pratiques traditionnelles - comme la création de palissades dans des virages étroits pour éviter que les chevaux et leurs jockeys se heurtent contre les murs.

Certaines villes et communautés ont cédé aux pressions et intégré ces nouvelles exigences, en célébrant leurs compétitions rituelles dans des circuits spéciaux construits à cet effet ou dans des stades adaptés pour l'occasion selon les normes prévues (comme à Legnano, par exemple). Néanmoins, ceci dépend pour beaucoup de la capacité économique des communautés à supporter le coût de ces aménagements, mais aussi de son adaptabilité culturelle afin de trouver un compromis entre respect des traditions et cérémonies et assimilation des normes, perçues comme extérieures et invasives.

Cela a été le cas du Palio de Sienne, qui a subi ces dernières années plusieurs modifications pour pouvoir continuer à être célébré. Les Palii d'Asti, de la Sartiglia de Oristano et d'autres compétitions équestres traditionnelles ont suivi le même modèle, en intégrant les nouvelles normes et sensibilités. Néanmoins, cette flexibilité n'est pas toujours aisée. Les Carresi du Bas Molise rencontrent plus de difficulté, en raison de la présence de bovins, qui complique encore davantage la question de l'acceptabilité éthique des compétitions rituelles et des risques qu'elles engendreraient pour les animaux impliqués. La première critique repose sur le fait que les bovins ne seraient pas des animaux «sportifs » et que ces courses représenteraient pour eux un effort et des risques trop graves de blessures ou simplement de stress. La deuxième critique est relative à la suspicion de dopage des animaux - en particuliers des bovins, afin d'améliorer leur vitesse - ces dernières années du fait de la tendance à la spectacularisation toujours plus grande des rituels. La troisième critique, enfin, est liée au fait que, pour guider et contenir la direction des courses de bovins, il est nécessaire de les pousser avec une lance pointue, qui se trouve désormais au cœur des attaques animalistes.

La visibilité de cette violence relative, existante dans ces courses - sang des blessures des animaux piqués par la lance, fractures lors des chutes - a suscité la cristallisation de la critique des mouvements animalistes, qui considèrent ces pratiques comme inacceptables au nom du bien-être animal.

Toutefois, aujourd'hui, au-delà même du droit animal, ce sont les normes de sécurité en matière de public qui deviennent prédominantes. Or, plus encore que la transformation des pistes de galop, elles exigent une reconceptualisation de ces pratiques patrimoniales et de l'imaginaire des compétitions historiques équestres. En effet, celles-ci passent d'un statut de traditions à fort caractère identitaire à un spectacle, devant en conséquence se soumettre à l'ensemble des règles de contrôle et des mises aux normes des grands événements publics. 

la commercialisation des droits d'images, qui exigent le respect des normes de sécurité, désormais visibles sur les images diffusées dans le monde entier. Dans ce cadre, le Palio de Sienne a connu ces dernières années une restriction drastique du nombre de spectateurs admis sur la place, à la suite d'événements tragiques qui ont changé la loi italienne ${ }^{4}$. Les voies d'évacuation sont également au cœur des enjeux de sécurisation de la place, mais demeurent compliquées à établir dans une ville ancienne organisée dans un dédale de rues, conçu pour favoriser l'entre-soi et non pour faciliter l'accès ou la sortie rapide. La mise en tourisme génère aussi des effets contradictoires, comme la gentrification d'une fête populaire, dont les prix d'accès ont connu ces dernières années une augmentation importante.

41 mais aussi économiques majeurs. En effet, ils ne sont plus uniquement des manifestations culturelles identitaires; ils représentent également des enjeux économiques cruciaux pour les villes concernées. C'est pourquoi, à la suite de la récusation de la candidature Unesco du Palio de Sienne, des polémiques ont éclaté au sein de la population locale. D'un côté, les représentants des Contrade (les partis/ quartiers citoyens qui se défient dans la course) soutenus par le Priorat des contrade (prieuré général des Contrade) proposaient de poursuivre leurs courses rituelles, en outrepassant les normes, même si cela signifiait l'absence de touristes et de support économique. De l'autre, les représentants de la ville, mais aussi des acteurs du tourisme et du commerce dans leur ensemble arguaient leur incapacité à pouvoir survivre à la disparition du Palio, un évènement désormais international devenu vital à leur équilibre économique. Ce conflit, qui a déchiré la population de Sienne, a engendré maints débats dans les médias locaux et au sein des instances des autorités locales. Il a de plus été relayé dans de nombreuses publications dans la presse locale (articles, appels, lettres de citoyens privés et d'autorités publiques, vignettes satiriques, etc.).

D'une façon générale, les organisateurs des Palii s'efforcent d'intégrer les nouvelles demandes pour éviter de disparaitre. Ainsi, à Legnano, par exemple, les médias ont beaucoup insisté sur l'effort consenti par l'organisation du Palio afin de sécuriser non seulement le public, mais surtout la santé et le bien-être des chevaux, grâce à tout un système de soins et de manifestations affectueuses envers l'animal, un fond de piste en sable pour la compétition et la réduction des actions coercitives et violentes (fouets, éperons, etc.) lors de la course. Malgré cela, les manifestations d'opposants se répètent, chaque année, devant le stade ou pendant le cortège historique qui se déroule avant la compétition traditionnelle.

Plus compliqué encore est le cas des Carresi, dont les parcours cérémoniels se déroulent tout au long des anciennes routes pastorales sur de nombreux kilomètres. Ces pratiques traditionnelles rencontrent de sérieux problèmes de mise aux normes, car il est économiquement difficile de recouvrir l'ensemble des chemins de sable et de sécuriser la totalité du parcours pour les publics... Mais leur suspension ou leur disparition ébranlerait les villes concernées, en récusant leur caractère patrimonial, ce qui fragiliserait l'identité partagée construite, mais aussi en déstabilisant leur économie actuelle et leur ouverture sur le monde. La photographie suivante, prise au cours d'une des manifestations de protestation contre l'arrêt des Carresi du Bas Molise en 2015 en raison du droit animal, en témoigne (cf. illustration 3). Sur cette photo, une affiche clame : « Vous nous avez volé histoire, présent et futur ». Cette affiche traduit la 
conscience locale de la blessure que représente pour les citoyens l'interdiction de célébrer leur fête à leur manière - remettant en question leur histoire - mais aussi l'importance de cet évènement pour leur développement et leur inscription dans un avenir. Cette affiche illustre également la plasticité des images patrimoniales, qui peuvent être utilisées, mises en forme, adaptées, critiquées et finalement absorbées dans le discours économique et politique contemporain.

Illustration 3. Ururi, 3 mai 2015

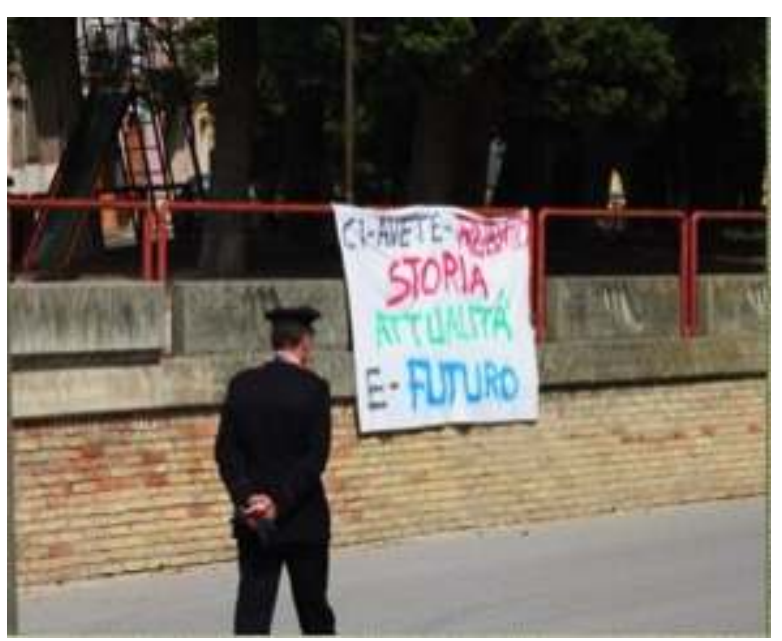

Source photo : Ballacchino, 2015

L'essor touristique de ces courses traditionnelles s'illustre, de la sorte, par un double phénomène d'apparence contradictoire.

Il exige, d'une part, un processus de normalisation et de sécurisation pour les animaux - plus particulièrement les chevaux - mais aussi pour les cavaliers et le public, ce qui peut fragiliser le caractère rituel de ces pratiques, en imposant une évolution des usages. Rappelons, en effet, que dans le passé ces cérémoniels se déroulaient au milieu de la foule, malgré le risque indubitable que cela pouvait représenter pour la population locale. Aujourd'hui, la transformation de la pratique en un événement touristique international majeur a généré de nouvelles préoccupations que les communautés ne comprennent pas ou ont du mal à gérer. Et ce, d'autant que cette touristification induit une mise en scène des pratiques cérémonielles, qui n'est pas toujours bien perçue par les habitants. Elle provoque aussi une confrontation des modalités techniques et des temporalités - temps longs de l'entrainement, des rituels de sacralisation - aux attentes des touristes, étrangers à cette culture, qui viennent seulement admirer le spectacle d'une course, sans toujours en comprendre les enjeux présents pour les communautés. En effet, la pression est très forte pour les jockeys et les Contrade, car les vainqueurs obtiennent le droit d'«humilier » les autres pendant un an. Le groupe vainqueur gagne la possibilité de porter en procession l'image du saint patron, de se moquer de ses adversaires en chantant des plaisanteries et de les taquiner pour leur incapacité et leur maladresse au cours de la course.

Néanmoins, le tourisme permet, d'autre part, à la fois un rayonnement national et international qui hisse les Palii au rang de patrimoine immatériel mondial - en dépit des contestations rencontrées - et des retombées économiques devenues vitales pour les villes, dont la renommée s'adosse désormais à ces évènements. 


\section{Conclusion}

À la lumière de notre analyse, nous pouvons conclure que le tourisme est un agent complexe pouvant permettre une perpétuation des courses équestres rituelles en Italie mais au travers d'un processus ambigu de recodification.

En effet, le système rituel et spectaculaire des compétitions traditionnelles équestres combine éléments identitaires et sociabilités importantes pour les communautés locales, et commercialisation nationale et internationale qui influence ses évolutions contemporaines. Si ce phénomène est exacerbé aujourd'hui, le caractère co-constitutif du tourisme et du patrimoine dans ces cérémonies, telles que le Palio ou les courses de bœufs et chevaux du Bas Molise, n'est pas nouveau puisque c'est dans ce contexte que ces évènements ont été codifiés dans la première moitié $\mathrm{du} \mathrm{xx}^{\mathrm{e}}$ siècle, afin de renforcer leur visibilité mais aussi de participer à leur pérennisation en les inscrivant dans un premier processus de normalisation. Le tourisme participe, de ce fait, à l'identification de ces cérémonies compétitives et, au-delà, à celle des villes organisatrices, qui se réinventent comme des "destinations » structurées autour d'une reconnaissance de pratiques traditionnelles élevées au rang de patrimoine immatériel (Lave et Wenger, 1991).

Le processus est néanmoins dynamique et en perpétuelle évolution. Ainsi, de nouveaux mouvements politiques interpellent transversalement les lieux communs liés à la notion de tradition, en mobilisant le droit des minorités, les questions de genre ou le droit animal. Dans ce contexte, les activistes du droit animalier ont entamé une procédure de déconstruction de la valeur patrimoniale de ces fêtes et cérémonies en les accusant d'être des signes d'une culture atavique, fondée sur un anthropocentrisme violent et qui doit être définitivement dépassé.

50 Cette volonté s'est traduite, dans le cas de Sienne, par la récusation ministérielle de la candidature Unesco du Palio. Dans d'autres cas, elle s'est illustrée par des conflits politiques et sociaux qui se déroulent autour des différentes compétitions traditionnelles avec animaux. Ainsi, les courses rituelles équestres en Italie cristallisent l'opposition de deux idéologies questionnant la notion de «patrimoine» et de «culture ». D'un côté, les activistes pour le droit animal condamnent ces pratiques, en récusant leur caractère patrimonial et en les accusant d'être des vestiges d'usages violents issus d'un autre temps, désormais folklorisés. De l'autre, des réactions, parfois virulentes également, des défenseurs de ces pratiques, qu'ils soient organisateurs ou communautés participantes, s'efforcent de démontrer l'importance, tant culturelle qu'identitaire et économique, du maintien de ces cérémonies pour les villes et villages concernés, car elles sont la principale source de dynamisation du commerce local, de l'élevage, mais aussi de la sociabilité des quartiers. Dans ce contexte, de nouvelles initiatives se font jour, comme l'amorce d'un nouveau dossier de candidature visant à faire reconnaître le Palio de Sienne comme patrimoine immatériel de l'Unesco, confié à une anthropologue par le ministère du Patrimoine culturel et du Tourisme italien, chargée d'identifier et de faire un inventaire de l'ensemble des savoir-faire et patrimoines culturels mobilisés par ce rituel.

51 La situation conflictuelle autour de ces compétitions rituelles permet de mettre en exergue, enfin, la confrontation entre des hommes parlant pour les hommes et des hommes parlant au nom de la « voix » des animaux, qui est au cœur des préoccupations

Mondes du Tourisme, 18 | 2020 
de l'anthropologie multi-espèces (Kohn, 2007), questionnant la subjectivité de chacun des acteurs - et le droit de penser "pour ", surtout dans le cas d'une autre espèce. Cet exemple nous permet aussi de comprendre ce genre d'activités communautaires dans un sens plus large et plus profond, et non seulement en tant que formes traditionnelles dépassées à caricaturer de manière simple et superficielle comme "sauvages et primitives ». Il est en effet nécessaire de dépasser ces oppositions trop binaires qui ne peuvent prendre en considération les échanges intersubjectifs ou formes de coopération entre espèces (Balcombe, 2009), permettant autant la survie d'une communauté qui s'identifie autour de ces rituels que celle des chevaux élevés à cet usage.

52 Ainsi, les traditions équestres représentent des «objets " privilégiés pour penser le rapport à la vie des humains contemporains en lien avec le monde animal et leur environnement, en termes à la fois de rituels et de systèmes producteurs d'identités et de développement économique, ainsi que de processus qui favorisent la socialisation au sein de la communauté (Ingold, 2000).

Cette réflexion nous conduit à une déconstruction utile de la rhétorique trompeuse des médias qui exploitent de façon souvent restrictive la notion de patrimoine en opposant des systèmes de valeur. Elle nous permet, également, de mettre en exergue le rôle complexe du tourisme qui, dans un double mouvement d'apparence contradictoire, favorise la perpétuation de ces patrimoines tout en exigeant des adaptations en termes de normes sécuritaires et de bien-être animal, qui nécessitent des reconceptualisations, parfois difficiles, tant d'un point de vue culturel qu'économique, pour les populations locales.

Le tourisme est donc un agent de déconstruction/reconstruction de ces traditions équestres recodifiées en patrimoine tendant à l'universel, qui interroge sur les modes de valorisation et de sauvegarde des patrimoines immatériels, en perpétuelle réinvention dans le présent.

\section{BIBLIOGRAPHIE}

Gerardo ADINOLFI, «Palio, la Brambilla ai toscani "Siena non è al centro del mondo" ", La Repubblica, 27 février 2017.

Arjun APPADURAI, «Introduction. Place and Voice in Anthropological Theory », Cultural Anthropology, vol. 3, n 1, 1988.

AA.VV., Immagine del Palio, Editore Nardini, 2003.

AA.VV., Visioni di Palio, Protagon Editori Toscani-Edizioni Alsaba, 2004.

Jonathan BALCOMBE, « Animal pleasure and its moral significance », Applied Animal Behaviour Science, $\mathrm{n}^{\circ}$ 118, 2009. 
Katia BALLACCHINO et Letizia BINDI, « Animals and/or Humans. Ethnography and Mediation of 'Glocal' Conflicts in the Carresi of Southern Molise (Italy) », dans Silvia PINTON et Lauso ZAGATO (dir.), Cultural Heritage. Scenarios 2015-2017, Venezia, 2017.

Katia BALLACCHINO et Letizia BINDI, « Tocca carriere... Note preliminari su una ricerca etnografica sulle Carresi del Basso Molise », Orma, $\mathrm{n}^{\circ}$ 22, 2014.

Luca BETTI et Alessandro FALASSI, Il Palio. La festa della città, Betti Editrice, 2003.

Letizia BINDI, «Cheval-patrimoine. Culture, territoire, économie d'une relation homme-animal », dans Beatriz SANTAMARINA (dir.), Geopoliticas patrimoniales. De culturas, naturalezas e inmaterialidades. Una mirada etnográfica, Germania : Collectiò Antropologiques, 2012.

Letizia BINDI, « A cavallo della tradizione: Palii, giostre e processioni lungo la penisola », Voci, IX, 2012.

Alessandra BROCCOLINI, «Intangible Cultural Heritage Scenarios within the Bureaucratic Italian State ", dans Regina BENDIX et al. (dir.), Heritage Regimes and the State, Universitatsverlag Gottingen, 2012.

Mauro civai et Enrico toti, Palio, la corsa dell'anima, Edizioni Alsaba, 1995.

Dona DAVIS, Anita MAURSTAD et Sarah COWLES, « Co-being and intra-action in horse-human relationships: a multispecies ethnography of be(com)ing human and be(com)ing horse ", Social Anthropology, vol. 21, n 3, 2013.

Wolfgang DRECHSLER, «The Contrade, the Palio and the Ben Comune: Lessons from Siena », Trames, vol. $10, \mathrm{n}^{\circ} 2,2006$.

Alan DUNDES et Alessandro FALASSI, La terra in Piazza: an interpretation of the Palio of Siena, University of California Press, 1975.

Alan DUNDES et Alessandro FALASSI, La terra in Piazza. Antropologia del Palio, trad. Italiana, Nuova Immagine Editrice, 2005.

Alessandro FALASSI, Per forza e per amore, Bompiani, 1980.

Roberto FILIANI et Natale ZAFFARONI, Con la Rivale in Campo 2, 1960-1989: le rivalità di contrada nelle carriere, nei numeri unici, nei racconti dei personaggi e le squalifiche negli atti dell'autorità comunale, Il Leccio, 2003.

Vincenzo GRASSI, Le Contrade di Siena e le loro feste - Il Palio attuale (vol. II: Il Palio sotto il Regno d'Italia 1859-1937: dal 1859 al 1900, dal 1901 al 1935, anni 1936-1937, più Cronistoria del Palio dal 1938 al 1972, de Alberto Tailetti), Edizioni U. Periccioli, 1972.

Stefan HELMREICH, Alien Ocean: Anthropological Voyages in Microbial Seas, University of California Press, 2009.

William HEYwOoD, Nostra donna d'agosto e il Palio di Siena, Protagon Editori Toscani, 1993.

Tim INGoLD, The Perception of the Environment. Essays on Livelihood, Dwelling and Skill, Psychology Press, 2000.

Eben KIRSKEY et Stefan HELMREICH (dir.), « The Emergence of Multispecies Ethnography », Cultural Anthropology, vol. 25, n 4, 2010.

Eduardo конN, « Hоw Dogs Dream. Amazonian Natures and the Politics of Transspecies

Engagement », American Ethnologist, vol. 34, n 1, 2007. 
Jean LAVE et Etienne WENGER, Situated Learning. Legitimate Peripheral Participation, Cambridge University Press, 1991.

Pietro MELONI, Il Tempo rievocato. Antropologia del patrimonio e cultura di massa in Toscana, Mimesis, 2014.

Fabio MUGNAINI, « Le feste neo-medievali e le rievocazioni stoeriche contemporanee tra storia, tradizione e patrimonio », Lares, vol. 79, n²-3, 2013.

Barbara NOSKE, Beyond Boundaries. Humans and Animals, Black Rose Books, 1997.

Jean-Pierre OLIVIER DE SARDAN, « La politique du terrain », Enquête, nº 1, 1995.

Paul RABINOW, « Artificiality and Enlightenment. From Sociobiology to Biosociality », dans John CRARY J et Stephen KWINTER (dir.), In-corporations, Zone, 1992.

Robert D. RYDER, Animal Revolution: Changing Attitudes towards Speciesism, Basil Blackwell, 1989.

Senio SENSI et Armando SANTINI, Guida al Palio, Siena, Betti Editrice, 1995-2002.

Susan SILVERMAN, « On the Use of History in Anthropology: The Palio of Siena », American

Ethnologist, vol. 6, n 3, 1979.

Alessandro simoniccA, « Le Contrade: uno sguardo antropologico », dans Pietro CLEMENTE P. (dir.), Contrada è..., Comitato Amici del Palio, 1990.

\section{NOTES}

1. Les Contrade du Palio de Siena sont au nombre de 17 au total depuis 1729 (Aquila, Bruco, Chiocciola, Civetta, Drago, Giraffa, Istrice, Leocorno, Lupa, Nicchio, Oca, Onda, Pantera, Selva, Tartuca, Torre, Valdimontone). Les conflits qui les opposent ont des origines très anciennes (remontant jusqu'au $\mathrm{x}^{\mathrm{e}}$ siècle), souvent associées à des questions de discordes entre territoires et familles dans la ville, avec des systèmes d'alliance entre certaines et des oppositions entre d'autres. À cause des dimensions de la Place del Campo, les Contrade qui courent à chaque édition du Palio ne peuvent être plus de dix. C'est pourquoi la sélection des Contrade qui vont courir à chaque édition est faite par tirage au sort, de même que la sélection du cheval qui représentera chaque Contrada (Filiani et Zafferoni, 2003).

2. Notre traduction.

3. Données de la chambre de commerce de Sienne 2018 et 2019. Voir : https://www.oksiena.it/ news/turismo-a-siena-nel-2019-in-crescita-arrivi-e-presenze-090420143855.html

4. En 2017, à Turin, la retransmission d'un match de football sur un écran géant dans l'une des places les plus importantes de la ville s'est transformée, en raison d'une échauffourée, en un moment de panique pour la foule, entraînant des blessures, de la peur et un décès. Cela a conduit à la publication d'un document très restrictif en matière de sécurité des manifestations publiques et des spectacles, la circulaire ministérielle dite « Gabrielli », du nom du directeur de la police qui l'a émise le 7 juin 2017, qui a été confirmée en 2018. 


\section{RÉSUMÉS}

Il existe en Italie de nombreuses manifestations traditionnelles très anciennes se concentrant autour de compétitions équestres - et animales, plus généralement. Certaines d'entre elles sont devenues des attractions touristiques très importantes à partir du début du $\mathrm{xx}^{\mathrm{e}}$ siècle.

Ces dernières années, un grand nombre de polémiques ont toutefois émergé contre ces pratiques rituelles compétitives, générant dans certains cas de grands débats, pouvant aller jusqu'à l'exclusion de toute forme de sauvegarde et de valorisation de caractère national ou global, ou encore à l'arrêt pur et simple de la pratique.

Cette situation nouvelle interpelle la réflexion scientifique sur les effets que peuvent avoir ces nouvelles sensibilités sur les cérémonies équestres traditionnelles, en questionnant leur influence sur la transformation de ces fêtes, qui deviennent des attractions touristiques.

Notre recherche repose sur une enquête ethnographique, combinant analyse de la littérature conceptuelle relative à l'ethnographie multi-espèces, étude de la littérature scientifique et des médias relatifs au Palio de Sienne (Toscane) et, enfin, observations et entretiens qualitatifs multisitués. Ces derniers se concentrent plus particulièrement sur les courses de chars à Carresi (BasMolise dans le sud de l'Italie) en 2012 et le Palio de Legnano (Lombardie) en 2017.

La recherche a permis de repenser les liens entre représentations et réalité des relations homme/ animal dans le cadre des manifestations traditionnelles, ainsi que les contradictions entre sensibilités contemporaines face aux pratiques culturelles locales qui deviennent de plus en plus des attractions touristiques, et de mettre en discussion le partage entre passé et présent, entre modernité et tradition à l'œuvre dans les contextes de promotion territoriale liés aux patrimoines immatériels.

There are many old traditional events in Italy focusing on equestrian (and animal, more generally) competitions. Some of them have been transformed into tourist attractions from the beginning of the 20th century. However, many controversies have recently arisen against these competitive ritual practices, generating in some cases major debates, which can go as far as the exclusion from the possibilities to submit these events, for example, to the 2003 UNESCO ICH List or even stopping outright from practice. This situation calls for scientific reflections on the effects of these new sensitivities on traditional equestrian ceremonies, by questioning their influence on the transformation of such as attractive festivals. The research is based on specific fieldworks, combining analysis of the conceptual literature relating to multispecies ethnography, scientific researches and different media productions relating to the Palio of Siena (Tuscany) and qualitative multi-site inquiry. The latter focus more particularly on the chariot races in Carresi (South-Molise in Southern Italy) in 2012 and the Palio de Legnano (Lombardy) in 2017. Methodology is focused on: interviews, focus groups, participant observation, shadowing and engagement techniques enabling to restore the complexity of the relationships between traditional equestrian practices, community building and tourism development in the regions concerned.

\section{INDEX}

Mots-clés : cérémoniels équestres, droit animalier, ethnographie inter-espèces, mise en tourisme, influence médiatique

Keywords : equestrian ceremonials, animal rights, inter-species ethnography, tourism, media influence 


\section{AUTEUR}

\section{LETIZIA BINDI}

Universitá degli Studi del Molise (Italie)

Anthropologue

letizia.bindi[at]unimol.it 